\title{
Avaliação da segurança, funcionalidade e qualidade das paradas de ônibus localizadas na cidade de Juiz de Fora
}

\author{
M. Salomão ${ }^{1 *}$, A. R. S. Oliveira ${ }^{1}$, R. C. Santos M. T. Barbosa ${ }^{1}$ \\ *Autor de Contato: mariana.salomao@engenharia.ufjf.br \\ ${ }^{1}$ Programa de Pós-graduação em Ambiente Construído, Faculdade de Engenharia, Universidade Federal de Juiz de \\ Fora, Juiz de Fora, Brasil
}

\section{RESUMO}

Com o crescimento das cidades, os transportes são indicadores do desenvolvimento sustentável, e suas instalações devem ser funcionais e proporcionarem qualidade e segurança aos usuários. $\mathrm{O}$ objetivo deste trabalho é efetuar uma vistoria técnica nos pontos de ônibus localizados na cidade de Juiz de Fora (MG/Brasil). Assim, efetuou-se uma avaliação qualitativa e quantitativa baseada numa revisão bibliográfica acerca das exigências mínimas a serem atendidas, analisando as paradas de ônibus da cidade. Os dados coletados avaliaram os requisitos de funcionalidade e segurança, bem como as manifestações patológicas identificadas e verificou-se uma elevada deficiência no que se refere à mobilidade urbana. Foram propostos serviços de manutenção corretiva, a fim de garantir a integridade do local, bem como a realização de adequações para garantir uma adequada mobilidade urbana para os usuários do transporte público local.

Palavras-chave: Transporte público; usuários; cidades sustentáveis; áreas urbanas; manifestações patológicas. 


\title{
RESUMEN
}

Con el crecimiento de las ciudades, el transporte es un indicador de desarrollo sostenible, y sus instalaciones deben ser funcionales y brindar calidad y seguridad a los usuarios. El objetivo de este trabajo es realizar una inspección técnica en las paradas de bus ubicadas en la ciudad de Juiz de Fora (MG / Brasil). Así, se realizó una valoración cualitativa y cuantitativa a partir de una revisión bibliográfica sobre los requisitos mínimos a cumplir, analizando las paradas de autobús de la ciudad. Los datos recopilados evaluaron los requisitos de funcionalidad y seguridad, así como las manifestaciones patológicas identificadas. Se han propuesto servicios de mantenimiento correctivo con el fin de garantizar la integridad del sitio, así como ajustes para asegurar una adecuada movilidad urbana para los usuarios del transporte público local.

Palavras-chave: Transporte público; usuarios; ciudades sostenibles; Areas urbanas; manifestaciones patológicas.

\begin{abstract}
With the growth of cities, transport is an indicator of sustainable development, and its facilities must be functional and provide quality and safety to users. The objective of this work is to carry out a technical inspection at the bus stops located in the city of Juiz de Fora (MG / Brazil). Thus, a qualitative and quantitative assessment was carried out based on a bibliographic review about the minimum requirements to be met, analyzing the city's bus stops. The collected data evaluated the functionality and safety requirements, as well as the pathological manifestations identified. Corrective maintenance services have been proposed in order to guarantee the integrity of the site, as well as adjustments to ensure adequate urban mobility for users of local public transport.
\end{abstract}

Palavras-chave: Public transport; users; sustainable cities; urban areas; pathological manifestations.

\section{INTRODUÇÃO}

O termo mobilidade urbana é definido como a condição que permite atender e suprir o deslocamento das pessoas em uma cidade para a realização das atividades do dia a dia como trabalho, educação, lazer, saúde, cultura, entre outros com o objetivo de desenvolver relações sociais e econômicas (Vaccari, 2011).

De acordo com os dados obtidos pelo Instituto de Pesquisa Econômica Aplicada (IPEA, 2016), a ocupação urbana no Brasil expandiu a partir de 1950, resultando num incremento de circulação de pessoas (Souza, 2017). Como o deslocamento das pessoas é diário e frequente é importante garantir que a mobilidade seja de qualidade, eficiente, segura e acessível, agregando benefícios a qualidade de vida da população (Antunes \& Simões, 2013). Entretanto, o Brasil possui um histórico deficiente no que se refere ao planejamento urbano rodoviário, gerando descontentamento aos cidadãos (Silva, 2021). Deve-se considerar, inclusive, a má qualidade do transporte público coletivo, sistema integrado na configuração da maioria das cidades brasileiras, responsável por cerca $95 \%$ dos deslocamentos urbanos (ANTP, 1997). 
Segundo Ferraz e Torres (2004) existem dez atributos necessários ao sistema de mobilidade por ônibus, a saber: acessibilidade, tempo de viagem, pontualidade, lotação, confiabilidade, características dos veículos, segurança, sistema de informação, comportamento dos operadores e características dos pontos de paradas; que são considerados na avaliação da qualidade dos serviços, já que a escolha do modal depende de suas características e a decisão final do usuário, procurando efetuar suas movimentações com qualidade e eficiência (Antunes e Simões, 2013).

Dentre os atributos acima mencionados, este trabalho destaca os pontos de ônibus, já que são uma área de integração entre os passageiros e o ônibus, sendo o primeiro contato entre eles (Andrade et al., 2004). Sendo assim, torna-se imprescindível instalações adequadas aos usuários.

Um PO ideal considera todos os aspectos quanto à: acessibilidade de pessoas com e sem deficiência; instalações adequadas para proteção contra intempéries, podendo esses abrigos contemplar apenas a cobertura ou, também, a cobertura com o fechamento lateral; bancos para os usuários; sinalização adequada; localização por questões de segurança a fim de se evitar acidentes; lixeira para promover a organização e limpeza do local; painel informativo; iluminação adequada (Brito et al., 2018; Filho, 2016;).

Nesse contexto, este trabalho tem por objetivo efetuar o levantamento dos PO localizados nas principais vias de acesso posicionadas nas 8 principais regiões que compõem a cidade de Juiz de Fora - Estado de Minas Gerais/Brasil identificando os parâmetros de mobilidade urbana, bem como efetuando um diagnóstico do estado de conservação dos mesmos a fim de propor estratégias de manutenção corretivas, seja pela garantia do ODS previsto pela ONU/2030 e/ou propiciar segurança da construção aos usuários, já que muitos se encontram em elevado estágio de degradação.

\section{MOBILIDADE URBANA}

\subsection{Considerações iniciais}

Devido ao intenso crescimento urbano no Brasil, a partir da década de 60, muitas cidades passaram a apresentar sistemas de mobilidade deficientes e com alto custo, com impactos negativos na vida das pessoas e nos custos econômicos e ambientais para a sociedade (Vasconcellos; Carvalho e Pereira, 2011). Portanto, o planejamento de transporte não pode ocorrer de forma isolada (Gouveia e Ferreira, 2011). A partir disso, a mobilidade urbana está sendo um dos maiores desafios da atualidade, tanto para o Brasil quanto para outros países, sendo previstas pela ONU como uma das metas a serem atendidas até 2030. Para o seu planejamento é preciso tornar, na atualidade, o fortalecimento da acessibilidade, nos níveis de serviços de transporte, de segurança, de fluidez e de qualidade ambiental (Filho, 2016).

No caso das cidades, o transporte coletivo é considerado um serviço essencial, tornando uma mobilidade mais acessível a todos (Araújo et al., 2011). Contudo, o que se tem observado nos últimos tempos é a transformação das cidades brasileiras em espaços eficientes para carros, cuja frota cresceu acentuadamente, através da ampliação do sistema viário e da utilização de técnicas de garantia de boas condições de fluidez (Andrade et al., 2004). Paralelo a isso, mesmo com alguns investimentos, o sistema de transporte público permanece insuficiente para atender à demanda da população, levando a um declínio da sua importância e tornando-se um "mal" necessário para a parcela da população que não tem acesso ao automóvel (Filho, 2016; Gouveia e Ferreira, 2011; Neto, 2004).

De acordo com a Associação Nacional das Empresas de Transportes Urbanos - NTU (2009) o transporte público coletivo é responsável pelo deslocamento de 59 milhões de passageiros/ dia 
sendo que o segmento de ônibus atende $92 \%$ da demanda. Portanto, é extremamente importante que esse método seja de qualidade.

\subsection{Pontos de ônibus (PO)}

Qualquer tipo de melhoria nas condições de operação do transporte público é sempre esperado pelos usuários, dentre eles, destaca-se, inclusive as benfeitorias nos PO (Andrade et al., 2004) que segundo a ABNT NBR- 15320 (2005) é definido como sendo o local onde os passageiros embarcam ou desembarcam na parada do veículo do transporte coletivo nas vias públicas, devendo atender a todos os cidadãos, inclusive os com deficiência. Trata-se, portanto, da conexão entre o sistema de circulação de pessoas com o sistema de transporte coletivo, o ônibus, sendo o elemento relevante na integração entre os dois sistemas (Carvalho, 2003). Possuem, dentre suas funções principais o conforto e a proteção às intempéries aos usuários do sistema de transporte (vide Figura 1), oferecendo assentos, disponibilizando maior segurança por meio das iluminações, painel informativo com representação das linhas, horários e itinerários, assim como lixeiras, localização dos pontos, incluindo ainda uma calçada adequada, para que todos tenham acesso ao ponto (Brito et al., 2018; Kneib et al., 2007). Assim como na Figura 1.

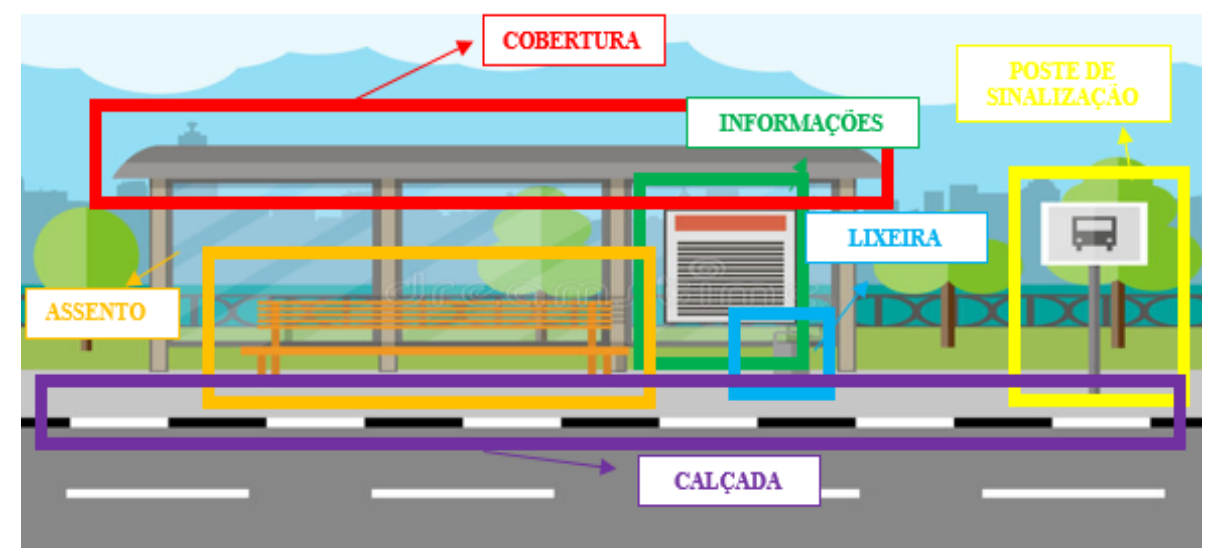

Figura 1. Parada do ônibus com as verdadeiras adaptações para as necessidades de bem-estar da população de Juiz de Fora - Minas Gerais. Fonte: Adaptada de Dreamstime.com. (Autor, 2021).

Pode se caracterizar por funcionalidade as informações apresentadas e que denota os aspectos positivos da interação entre um indivíduo e o meio externo, sendo elas denominadas como principais manifestações patológias as perspecções da população, pretendendo haver constantemente a necessidade de segurança em avaliação de qualificáveis construções das calçadas, ponto de sinalização, assim como a iluminação para o seu uso, pois segurança se denomina por demonstração total da certeza sobre uma convicção ao afastamento do perigo, uma boa cobertura, com assentos prioritários na utilização dos PO em prol da qualidade da infraestrutura que preconiza uma indispensável edificação de eficiência aos seus usuários, assim como uma calçada de fácil acessibilidade, iluminação para segurança, e as informações dos painéis de uso coletivo diante as informações essenciais diante a sinalização.

\section{METODOLOGIA}

A cidade de Juiz de Fora está localizada na região da Zona da Mata do estado de Minas Gerais, Brasil e possui uma população, segundo o censo do Instituto Brasileiro de Geografia e Estatística 
de 2010, estimada em 516.547 habitantes distribuídos numa área de $1.435 .749 \mathrm{~km}^{2}$. A sua localização estratégica possibilita um contato com os maiores mercados consumidores do país, Rio de Janeiro e São Paulo, conforme ilustrado na Figura 2.

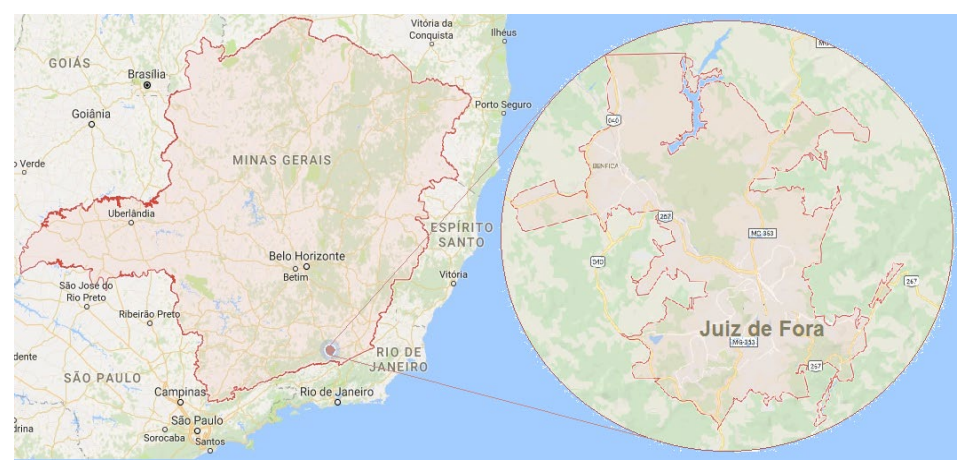

Figura 2. Localização da cidade de Juiz de Fora. Fonte: Adaptado de Google, 2017.

A cidade é dividida em oito regiões de acordo com o mapa mostrado na Figura 3, e cada uma dessas regiões é composta por diversos bairros. Nesse sentido, esse estudo efetuou o levantamento de dados (funcionalidade, acessibilidade, segurança, incluindo nesse último o levantamento das anomalias) nos PO nas ruas principais de acesso a cada uma das regiões, a saber: Centro: Avenida Rio Branco (constituída por 37 POs); Oeste: Avenida Deusdedith Salgado (constituída por 20 POs); Leste: Avenida Sete de Setembro (constituída por 7 POs); Nordeste: Rua Paracatu (constituída por 9 POs); Sul: Rua Ibitiguaia (constituída por 9 POs); Norte: Avenida Presidente Juscelino Kubitschek (constituída por 65 POs); Sudeste: BR-267 em seu perímetro urbano (constituída por 14 POs); Noroeste: Avenida Brasil (constituída por 32 POs).

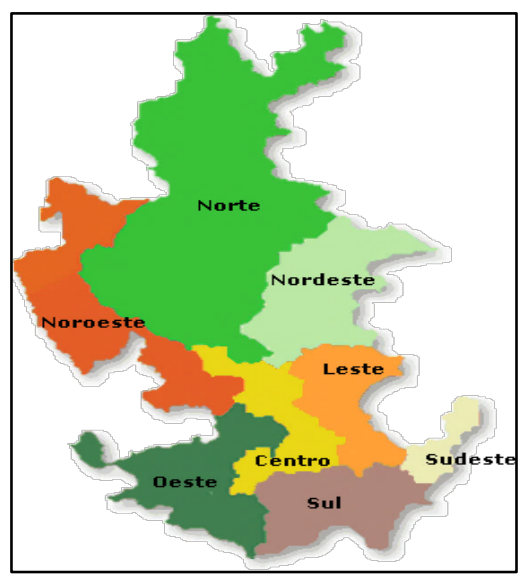

Figura 3. Regiões da cidade de Juiz de Fora. Fonte: Acessa.com, 2017.

Para o desenvolvimento do trabalho foram utilizados alguns dos atributos sugeridos por Ferraz e Torres (2004) mencionados anteriormente juntamente com as identificações das variáveis sugeridas por Filho (2016).

O estudo consistiu além da revisão bibliográfica a coleta de dados em campo efetuado por meio de análise visual e registro iconográfico (fotografia) dos principais itens que serão analisados. A metodologia analisada permitiu identificar e mapear as principais falhas, e a partir disso, propor soluções estratégias de manutenção corretivas contidas na bibliografia, possibilitando a realização de adequações a fim de propiciar uma adequada mobilidade urbana para os usuários do transporte público local. 


\section{RESULTADOS E ANÁLISES}

Salienta-se que foi adotado como padrão de classificação dos itens analisados diante a um levantamento de dados com o objetivo de efetuar o levantamento dos PO (pontos de ônibus) localizados nas principais vias de acesso posicionadas nas 8 principais regiões que compõem a cidade de Juiz de Fora - Estado de Minas Gerais/Brasil identificando os parâmetros de mobilidade urbana (Tabela 1 e Gráfico 1), bem como efetuando um diagnóstico do estado de conservação (Tabela 2 e Gráfico 2) dos mesmos a fim de propor estratégias de manutenção corretivas.
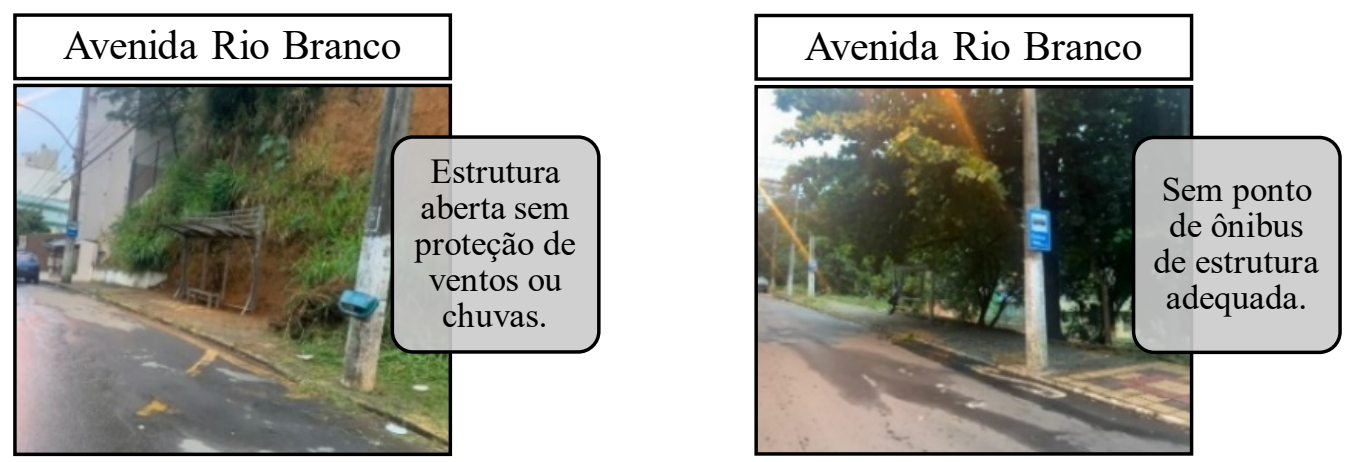

Figura 4 - Região Central: Avenida Rio Branco. Fonte: Autores, 2021.
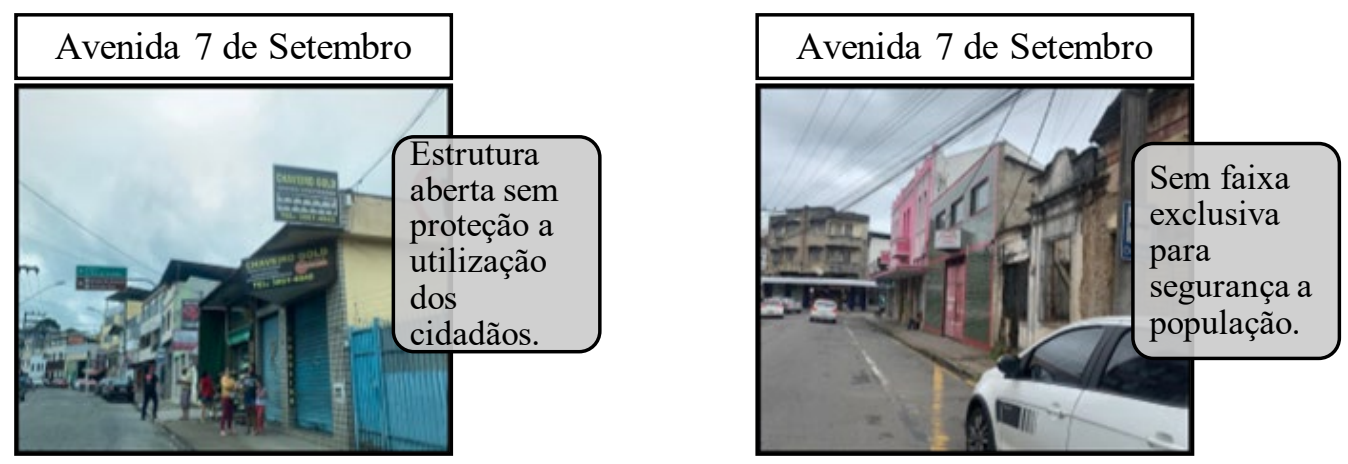

Figura 5 - Região Oeste: Avenida 7 de Setembro. Fonte: Autores, 2021.
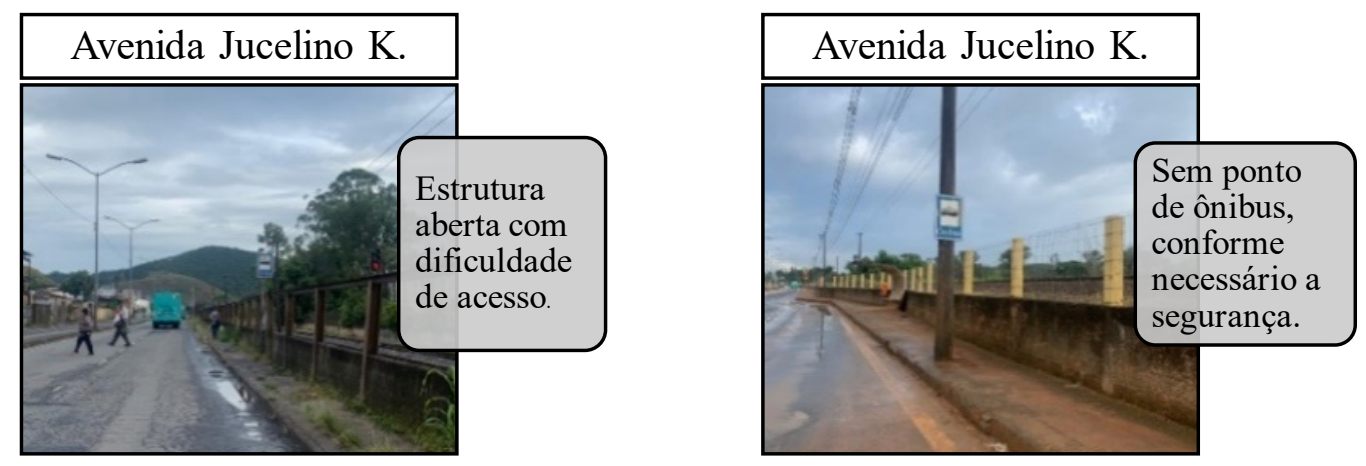

Figura 6 - Região Leste: Fonte: Autores, 2021. 

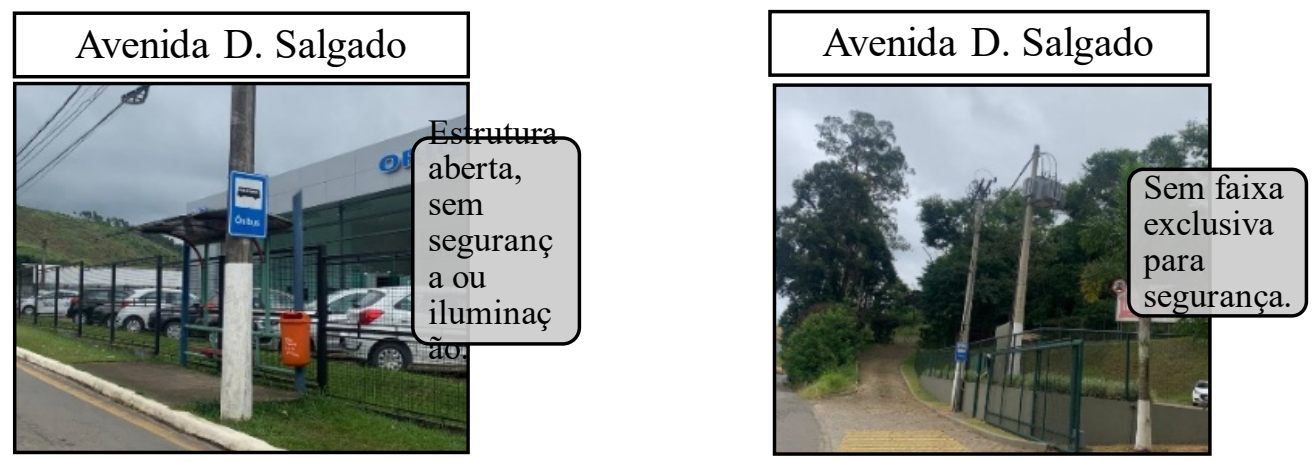

Figura 7 - Região Nordeste: Avenida D. Salgado. Fonte: Autores, 2021.
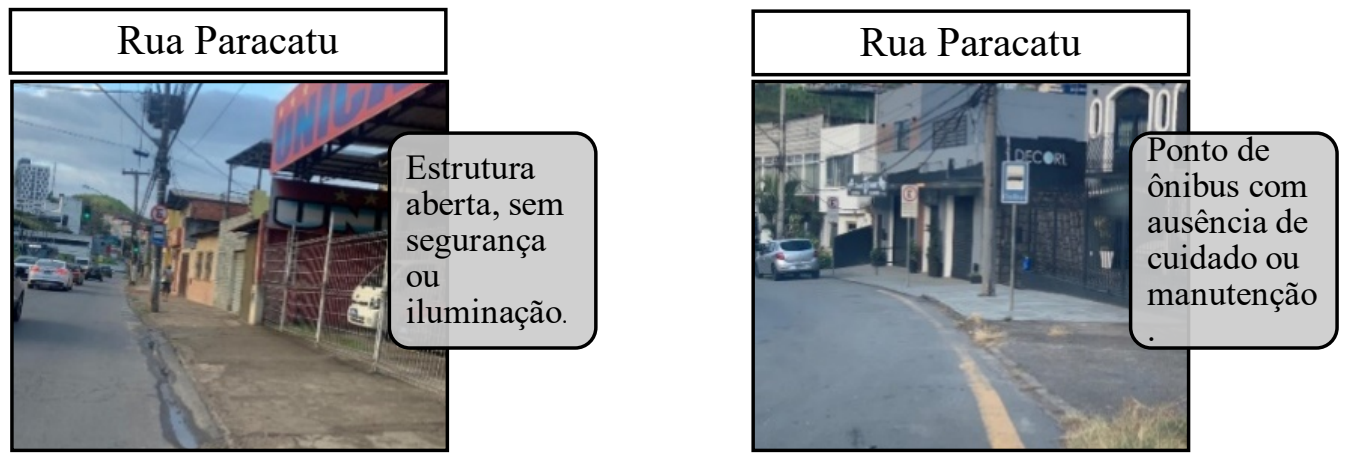

Figura 8 - Região Sul: Rua Paracatu. Fonte: Autores, 2021.
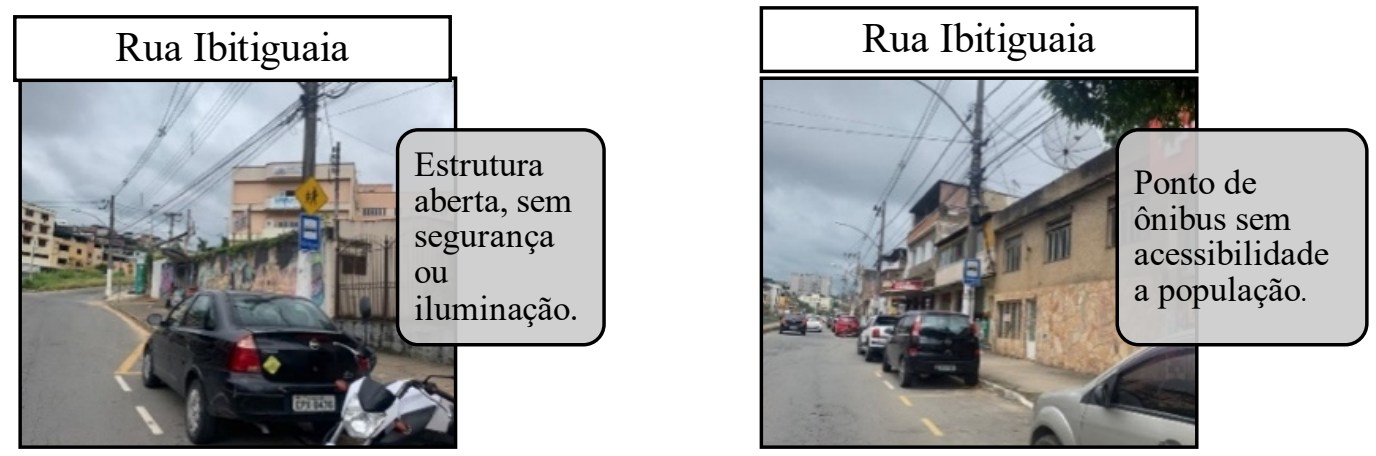

Figura 9 - Região Norte: Rua Ibitiguaia. Fonte: Autores, 2021.
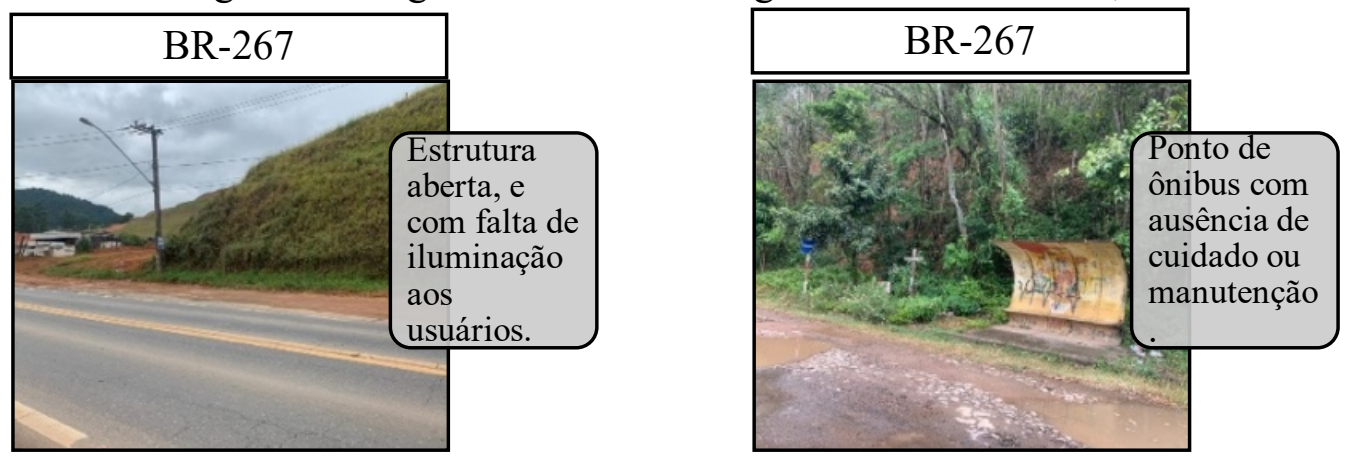

Figura 10 - Região Sudeste: BR-267. Fonte: Autores, 2021. 

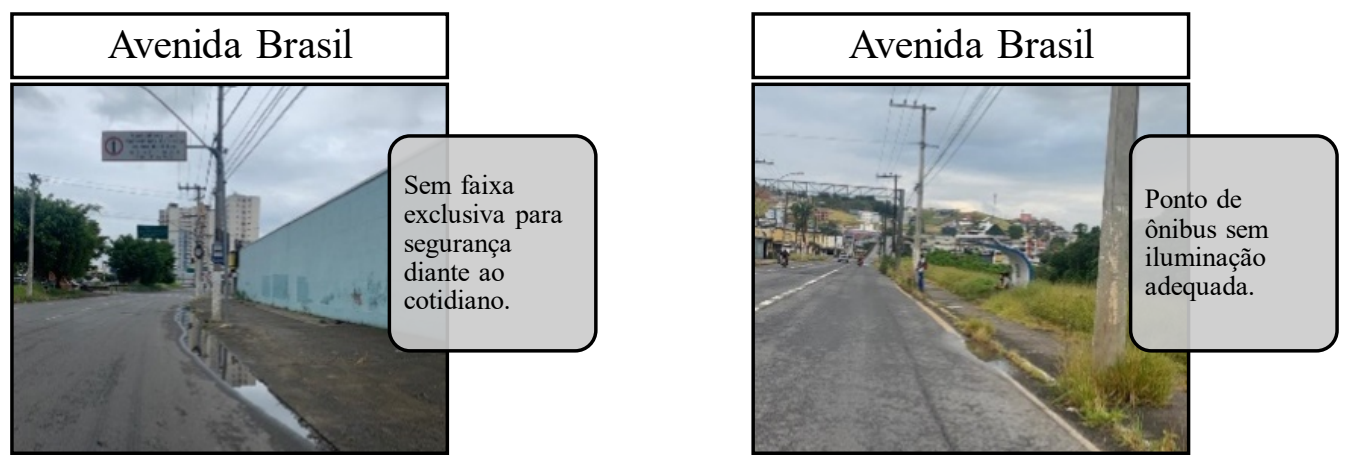

Figura 11 - Região Noroeste: Avenida Brasil. Fonte: Autores, 2021.

Tabela 1. Levantamento dos aspectos essenciais a mobilibilidade urbana por região.

\begin{tabular}{|l|l|l|l|l|l|}
\hline \multicolumn{1}{|c|}{ Região } & Acessibilidade & Funcionalidade & Segurança & Sinalização & Infraestrutura \\
\hline Centro & & & & & \\
\hline Oeste & & & & & \\
\hline Leste & & & & & \\
\hline Nordeste & & & & & \\
\hline Sul & & & & & \\
\hline Norte & & & & & \\
\hline Sudeste & & & & & \\
\hline Noroeste & & & & & \\
\hline
\end{tabular}

Gráfico 1 - Mobilidade urbana em Juiz de fora e seus aspectos em meio a um total de 193 PO.

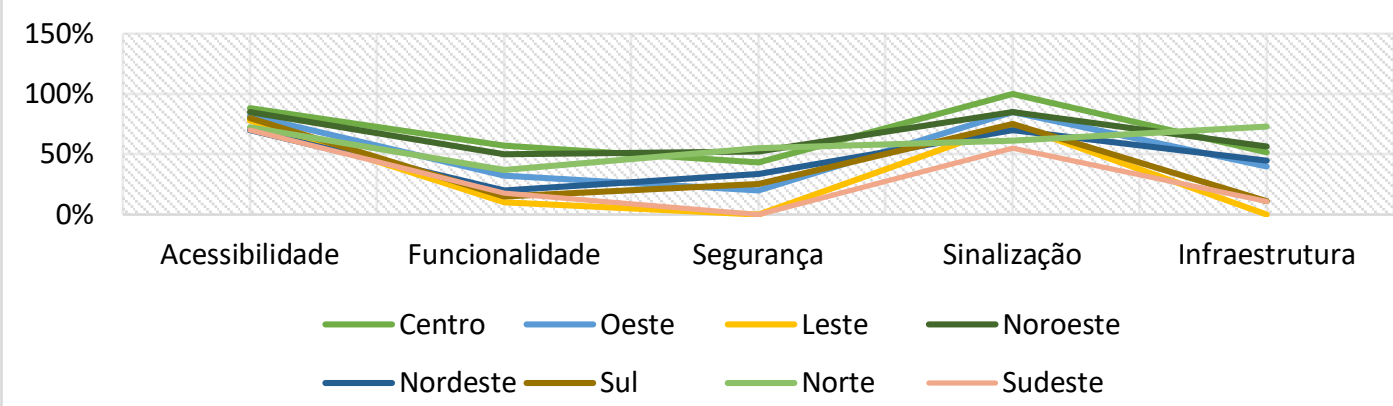

Analisando o Gráfico 1, verifica-se uma elevada deficiência nos pontos de ônibus (PO) no que se refere à mobilidade urbana apesar da cidade ser reconhecida por possuir bons índices de qualidade de vida. Apresentando mediante as consistências do gráfico com um percentual aproximado conforme verificado por região, com 193 pontos de ônibus no total para abranger os conteúdos do projeto, mas divididos por regiões em meio as funcionalidades do cotidiano: Centro: Avenida Rio Branco, com um total de 37 pontos de ônibus; Oeste: Avenida Deusdedith Salgado, com um total de 20 pontos de ônibus; Leste: Avenida Sete de Setembro, com 7 pontos de ônibus; Noroeste: Avenida Brasil, com um total de 32 pontos de ônibus; Nordeste: Rua Paracatu, com 9 pontos de ônibus; Sul: Rua Ibitiguaia, com 9 pontos de ônibus; Norte: Avenida Presidente Juscelino Kubitschek, com 65 pontos de ônibus; Sudeste: BR-267 em seu perímetro urbano, com 14 pontos de ônibus distintos.

No que se refere as manifestações patológicas constata-se que diante aos 193 pontos de ônibus (PO), no total, e subvidido por região, constata-se (vide Tabela 2 e Gráfico 2) a necessidade de 
desenvolver estratégias de manutenção corretiva. Cosnta-se que as regiões com boa acessibilidade e bom funcionamento são a central (Avenida Rio Branco) e a Noroeste (Avenida Brasil), sendo necessário ao órgão responsável implementar serviços de manutenção mais eficazes nas demais em prol da garantia da boa mobilidade urbana, assim como nas capacitações ainda mais eficazes para suas manifestações patológicas diante aos resultados necessários de longo prazo a seus métodos de desenvolver boas resoluções construtivas aos PO.

Tabela 2. Levantamento dos aspectos patológicos diante a mobilibilidade urbana por região.

\begin{tabular}{|l|l|l|l|l|l|l|}
\hline Região & $\begin{array}{c}\text { Buracos na } \\
\text { calçada }\end{array}$ & Fissuras & $\begin{array}{c}\text { Carpinagem } \\
\text { das gramas }\end{array}$ & Vandalismo & $\begin{array}{c}\text { Ausência } \\
\text { de assento }\end{array}$ & $\begin{array}{c}\text { Ausência } \\
\text { de toldo }\end{array}$ \\
\hline Centro & & & & & & \\
\hline Oeste & & & & & & \\
\hline Leste & & & & & & \\
\hline Nordeste & & & & & & \\
\hline Sul & & & & & & \\
\hline Norte & & & & & & \\
\hline Sudeste & & & & & & \\
\hline Noroeste & & & & & \\
\hline
\end{tabular}

Gráfico 2 - Os percentuais diante as necessidades de manutenções em Juiz de Fora (MG).

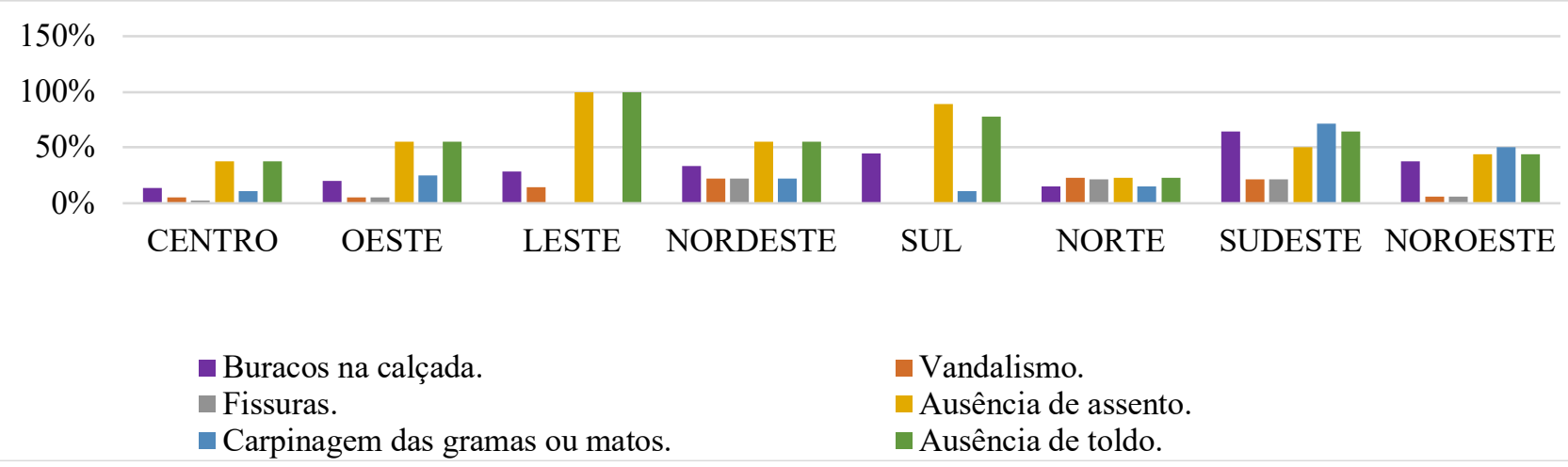

Nos resultados obtidos mediante a pesquisa feita de 193 PO no total, analisamos em meio as anomalias, conforme a mobilidade urbana, que para existir de forma qualificável a se movimentar e a exister bem-estar de vida, os PO existentes na cidade de Juiz de Fora devem possuir manutenções preventivas, pois são importantissímas à medida em que a edifícação vai ficando conforme o tempo mais desgastada, de uma idealização fortemente garantida para um maior índice de satisfação em meio ao desempenho estrutural das manutenções adequadas diante aos buracos na calçada, as fissuras diante o concreto ou aço armado, assim como para os vandalismos, assentos quebrados e de infraestrutura que comprometa significamente a acessibilidade e segurança da população. Sendo essencial e unicamente sempre buscarmos bons materiais para as distintas etapas das construções de cada edificação realizada de determinados projetos elaborados, e que os mesmos sejam de eficiência durante os desenvolvimentos das contruções para que existam perante as necessidades de Juiz de Fora um desempenho estrutural eficaz diante o uso diário da população, com precarizações a manutenções regularmente realizadas em meio aos seus atendimentos em prol a acessibilidade urbana, corraqueiramente da avaliação e funcionalidade local. 


\section{CONCLUSÃO}

A mobilidade urbana reconhecida pela cidade de Juiz de Fora de denominação a ser o principal objeto de estudo, pode-se verificar que a mobilização vem sendo a forma pela qual as pessoas realizam sua locomoção de forma frequente, sendo de grande importância garantir que esta mobilidade seja de qualidade, eficiente, segura e acessível, o que irá resultar em qualidade de vida da população. Entretanto, o Brasil tanto antigamente como ainda hoje, possui um histórico de planejamento urbano rodoviário muitas vezes sem o aperfeiçoamento estrutural, de acessibilidade e de capacidade em meio aos $\mathrm{PO}$, ocasionando deficiências no que se refere à mobilidade urbana e, consequentemente, gerando insatisfações diante à sociedade.

Deve-se considerar, inclusive, que objetos do mobiliário urbano devem proporcionar aos cidadãos sempre bem-estar e qualidade em suas mediações, sendo os mais comuns no Brasil. Os referenciais em meio a seus abrigos, que tem como função dar conforto e proteção as intempéries aos usuários do sistema de transporte localizados na cidade de Juiz de Fora, podem contemplar somente a cobertura ou também a cobertura com o fechamento lateral, e devem oferecer assentos para que os usuários possam aguardar a chegada do transporte coletivo com iluminação para aumentar a segurança da população, painel informativo com informações referentes aos ônibus, linhas, horários e itinerários para melhor atender a população e melhor equilibrar o tempo diante as suas utilizações. Onde as manifestações patológicas do andamento da construção civil podem se originar em qualquer uma de suas etapas, na qual um tal fator nota-se a importância do controle adequado, padronização para conforte e qualidade na execução dos serviços que constituem o processo como um todo para que nao agravem significamente nas patologías estruturais.

Por isso, a grande importância dos ínumeros métodos de controle na qualidade e de referências normativas para assegurar devem garantir altos níveis de desempenho, vida útil e durabilidade de edifícios são fundamentais, determinando no seguinte trabalho quis proporcionar e refletir aos leitores o verdadeiro significado de melhoria em meio a mobilidade urbana, pelas suas grandes deformidades em qualidade, bem-estar, precarização, principalmente nos últimos tempos com o alto índice do crescimento urbano dentro e fora do território brasileiro. Isso foi possível através da análise de documentos, entrevistas e questionários, demonstrando a existência de uma visão muito negativa da realidade da mobilidade urbana da cidade, a precarização e falta de cuidado em meio aos gestores de organização e de responsabilidades governamentais diante a empreendimentos gerenciados em destaque da qualidade de vida em Minas Gerais ao preconizar estratégias corretivas de caráter assertivo em segurança, exclusividade em pertinência a pontos de ônibus ou estações de transportes como em acessibilidade a população, mediante aos materiais, projetos, cronograma, além das qualidades das construções diante a algumas intermediações dos órgãos responsáveis a um bem-estar a população, por meio da avaliação da segurança, funcionalidade e qualidade das paradas de ônibus localizadas na cidade de Juiz de Fora.

\section{AGRADECIMENTOS}

CNPQ - Conselho Nacional de Desenvolvimento Científico e Tecnológico - Brasil

CAPES - Coordenação de Aperfeiçoamento de Pessoal de Nível Superior 


\section{REFERÊNCIAS}

Almeida, E.; Giacomini, L.; Bortoluzzi, M. Mobilidade e acessibilidade urbana. Seminário Nacional de Construções, 2013. Disponível em: https://www.imed.edu.br/Uploads/Mobilidade $\% 20 \mathrm{e} \% 20$ Acessibilidade $\% 20$ Urbana.pdf. Acesso em: 29 Jan. 2021.

Andrade, K.; Paula, V.; Mesquita, A.; Vilella, P. Problemas relacionados aos pontos de parada do transporte público nas cidades de porte médio. Instituto de Engenharia de São Paulo, p. 1-7, 2004. Disponível em: $<$ http://lares.org.br/Anais2004/trabalhos/G/Problemas\%20relacionados\%20aos\%20pontos\%20de \%20parada.pdf $>$. Acesso em: 27 jan. 2021.

Antunes, E.; Simoes, F. Engenharia urbana aplicada: um estudo sobre a qualidade do transporte público em cidades médias. URBE. Rev. Bras. Gest. Urbana, Curitiba, v. 5, n. 2, p. 51-62, Dec 2013. Disponível em: $<$ http://www.scielo.br/scielo.php?script=sci_arttext\&pid=S217533692013000200006\&lng=en\&nrm=iso $>$. Acesso em: 16 jan. 2021.

Araújo, M.; Oliveira, J.; Jesus, M.; Santos, P. A.; Lima, T. C. Transporte público coletivo: discutindo acessibilidade, mobilidade e qualidade de vida. Psicologia \& Sociedade, v. 23, n. 3, p. 574-582, 2011.

Associação Brasileira de Normas Técnicas. (2005). NBR 15320: Acessibilidade à pessoa com deficiência no transporte rodoviário. Rio de Janeiro.

Associação Nacional de Transporte Público (1997). Transporte Humano. Cidades com qualidade de vida.

Associação Nacional Das Empresas de Transportes Urbanos (2009). Desoneração dos Custos das Tarifas do Transporte Público Urbano e de Característica Urbana.

Balassiano, B. (1997). Planejamento Estratégico de Transportes Considerando Sistemas de Média e Baixa Capacidade. In: Congresso de Pesquisa e Ensino em Transportes - ANPET, 11, Rio de Janeiro, 1997. Anais.v.1, p.203-216.

Brito, A.; Santos, J..; Gonçalves, M. A.; Marques, R.; Fontana, A. M. Estudo sobre as condições das paradas de ônibus na cidade de São Paulo. 2018. Disponível em: https://docs.google.com/viewerng/viewer?url=https://ojs.eniac.com.br/index.php/EniacPesquisa/a rticle/viewFile/495/pdf. Acesso em: 18 jan. 2021.

Carvalho, E. B. Indicadores de acessibilidade no entorno de paradas de ônibus: proposta de classificação em níveis de serviço. 2003. Tese de Doutorado. Dissertação de mestrado em Transportes. Universidade de Brasília.

Confederação Nacional de Transporte (2002). Avaliação da operação dos corredores de transporte urbano por ônibus no Brasil.

Costa, J.; Santos, V. (2006). O desenvolvimento do sistema integrado de transporte coletivo de Aracaju e suas contradições. Candeeiro, 9, 77-83. 
Cruz, T. (2020). Você sabe o que é mobilidade urbana e qual o seu impacto na arquitetura?. VivaDecora PRO.

EDUCAMAIS. Capacitação profissional: o que é, importância e principais desafios. 2020. Disponível em: $<$ https:/www.educamaisbrasil.com.br/educacao/carreira/capacitacao-profissionalo-que-e-importancia-e-principais-desafios.> Acesso em: 16 jan. 2021.

Ferraz, A.; Torres, I. Transporte público urbano. São Carlos: Rima, 2004. In: ANTUNES, E. M.; SIMOES, F. A. Engenharia urbana aplicada: um estudo sobre a qualidade do transporte público em cidades médias. URBE. Rev. Bras. Gest. Urbana, Curitiba, v. 5, n. 2, p. 51-62, Dec 2013. Disponível em: $\quad<$ http://www.scielo.br/scielo.php?script=sci_arttext\&pid=S217533692013000200006\&lng=en\&nrm=iso>. Acesso em: 16 jan. 2021.

Filho, R. B. Análise da percepção das condições da infraestrutura dos pontos de ônibus oferecida aos usuários: Um estudo de caso da Cidade de São João da Boa Vista, SP, 2016. Disponível em: https://repositorio.ufscar.br/bitstream/handle/ufscar/8020/DissRBF.pdf?sequence=1\&isAllowed= y. Acesso em: 18 jan. 2021.

Gouveia, C. F.; Ferreira, W. R. (2011). Análise do transporte público coletivo em pequenas cidades - Tupaciguara e Sacramento/ MG. Horizonte Científico.

Instituto de Pesquisa Economica Aplicada. Sistema de indicadores de percepção Social (SISPS): Mobilidade urbana. Brasília, 2011. Disponível em: $<$ http://www.ipea.gov.br/portal/images/stories/PDFs/SIPS/110124_sips_mobilidade.pdf $>$. Acesso em: 25 jan. 2021.

Instituto Brasileiro de Desenvolvimento Da ArquiteturA. O que são patologias de construção ?. Fórum da Construção. Fonte:www.ibape-rs.org.br, 2021. Disponível em: http://www.forumdaconstrucao.com.br/conteudo.php?a=17\&Cod=1620

Kneib, E. C.; Morais; A.C de; Silva; P. C. M. da; Aragão, J. G. de. Contribuição metodológica para avaliação da acessibilidade a pontos de parada de transporte coletivo: estudo aplicado ao Distrito Federal. 2007.

MJF - Disponível em: https://pt.mapsofworld.com/where-is/juiz-de-fora.html. Acesso em 16 de Fevereiro.

MOOVIT. Aplicativo de Mobilidade urbana. 2021. Disponível em: https://moovitapp.com/index/pt-br/transporte_p\%C3\%BAblico-lines-Juiz_de_Fora-2752-851736. Acesso em 26 jan. 2021.

NACTO. National Association of City Transportation Officials. New York, ORG.

Neto, O. L. Um novo quadro institucional para os transportes públicos: condição sine qua non para a melhoria da mobilidade e acessibilidade metropolitana. 2004. In E. Santos \& J.Aragão (Orgs.), Transporte em tempos de reforma: estudos sobre o transporte urbano (pp. 193-216). Natal: EDUFRN.

Pires, A. C. M.; Pires, L. R. G. M. (orgs). Mobilidade Urbana desafios e sustentabilidade. 2016. Ponto e Linha. 1a ed. São Paulo. 2016. Disponível em: http://cidadeemmovimento.org/wp- 
content/uploads/2016/10/Mobilidade-Urbana-Desafios-e-Sustentabilidade.pdf. Acesso em: 16 jan. 2021.

QUALIONIBUS. Transporte coletivo de qualidade - QualiOnibus. WRI Brasil, São Paulo.

Silva, A. N. R. da; Ferraz, A.C. P. (1991). Transporte público urbano: operação e administração. [S.l: s.n.].

Silva A. P., Jonov C.M.P. Curso de especialização em construção civil. Departamento de engenharia de materiais e construção. Minas Gerais, 2011. (Notas de Aula). Disponível em: $<$ http://www.demc.ufmg.br/adriano/Manifest_\%20Pat_2016.pdf $>$. Acesso em: 06 nov. 2016.

Silva, J. C. da. A estratégia brasileira de privilegiar as rodovias em detrimento das ferrovias; Brasil Escola. 2021. Disponível em: https://brasilescola.uol.com.br/geografia/por-que-brasiladotou-utilizacao-das-rodovias-ao-inves-.htm. Acesso em: 18 jan. 2021.

Souza, D. B. de. Mobilidade urbana como instrumento de desenvolvimento sustentável. Âmbito Jurídico. 2017. Disponível em: https://ambitojuridico.com.br/cadernos/direitoadministrativo/mobilidade-urbana-como-instrumento-de-

desenvolvimentosustentavel/\#: :text $=\mathrm{A} \% 20$ mobilidade $\% 20$ urbana $\% 20$ pode $\% 20$ ser,com $\% 20$ ofer ecimento\%20n\%C3\%A3o\%20s\%C3\%B3\%20de. Acesso em: 25 jan. 2021.

VaccarI, L. S.; Fanini, V. Mobilidade urbana. Série de Cadernos Técnicos, Conselho Regional de Engenharia, Arquitetura e Agronomia do Paraná (CREA-PR), Paraná, 2011. Disponível em: $<$ https://www.crea-pr.org.br/ws/wp-content/uploads/2016/12/mobilidade-urbana.pdf $>$. Acesso em: 29 Jan. 2021.

Vaiano, P. Pesquisa: brasileiros passam mais de duas horas por dia no trânsito. Publicado em 31/05/2019 por Revista AlgoMais às 5:30 - Brasil. Disponível em: http://revista.algomais.com/urbanismo/pesquisa-brasileiros-passam-mais-de-duas-horas-por-diano-transito

Vasconcellos, E. A. de; Carvalho, C. H. R. de; Pereira, R. H. M. Transporte e mobilidade urbana. 2011. Disponível em: < http://repositorio.ipea.gov.br/bitstream/11058/1373/1/TD_1552.pdf>. Acesso em: 30 Jan. 2021.

Vaz, J. C; Santoro, P. (2009). Cartilha Mobilidade urbana é desenvolvimento urbano! 2005. Disponível em: $<$ http://www.polis.org.br/publicacoes_interno.asp?codigo=1>. Acesso em: 30 Jan. 2021. 